\title{
Cognitive Agent Based Identification of Relevant Auctions in Mobile E-Commerce
}

\author{
Nandini S. Sidnal ${ }^{{ }^{*}}$, Sunilkumar S. Manvi ${ }^{2}$ \\ ${ }^{1}$ Department of Computer Science and Engineering, KLE Society's College of Engineering and Technology, Belgaum, India; \\ ${ }^{2}$ Department of Electronics and Communication Engineering, Wireless Information Systems Research laboratory, REVA Institute of \\ Technology and Management, Bengaluru, India. \\ Email: sunil.manvi@revainstitution.org, sidnal.nandini@gmail.com
}

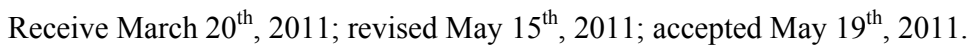

\begin{abstract}
The increased attention of E-auction services in mobile E-commerce demands an approach to identify the relevant auctions as per the bidder's requirements so as to increase the bidder satisfaction level and auction winning probability. In this paper, we propose an intelligent agent based model to identify the relevant set of auctions for a mobile bidder based on the bidder's requirements, preferences and constraints from a set of active auctions available in the active auction service directory in regional gateway connected to Internet. The agent functions are based on Belief, Desire and Intention $(B D I)$ cognitive architecture and are capable of taking dynamic decisions to search the matching auctions for the products requested by the bidder in the bidder belief set located in the bidder's mobile device and/or in the case base of the regional gateway. If matching auctions are not found in either of them, BDI agent searches them in the active auction service directory and computes the relevance factor based on the parameters in the bidder's requirements for all the matching active auctions and clusters into relevant (or potential) and non relevant auctions. The model is simulated to test the performance measures like availability of relevant auctions, average response time, and probability of winning auctions with better satisfaction. The proposed model is also compared with advertisement based auction service discovery model to show its effectiveness.
\end{abstract}

Keywords: Auctions, BDI Agents, Mobile E-Commerce, K-Means Clustering, Case Based Reasoning, Relevant Auctions

\section{Introduction}

Mobile E-commerce is an emerging area of E-commerce that is defined as buying and selling of services, information or goods without any location restrictions, using mobile device which use wireless connections to establish communication between all necessary parties to complete the transaction. Mobile E-Auctions is one of the popular applications of M-commerce [1] which is gaining popularity due to their support to buy and sell goods ubiquitously using wireless handheld devices. This ubiquitous feature has caused enormous increase in number of Eauction services and hence selecting the appropriate set of E-auction services manually to participate in bidding is becoming a challenging task. Hence a mechanism that provides autonomy, reliability and intelligent ability to deal with dynamic situations to identify the relevant set of auction services as per the bidder's requirements and preferences is to be devised. To devise such a mechanism, software agent paradigm comprising intelligent static and mobile agents can be adopted for E-business applications
$[2,3]$.

A software agent is a computer program which works towards goals in a dynamic environment on behalf of another entity (human or computational) without continuous direct supervision or control and exhibits a significant degree of flexibility and even creativity in how it seeks to transform goals into action tasks. These agents can be static or mobile in nature. But both of them perform the assigned tasks intelligently. Functionally and logically both perform in the same way except that mobile agents can be programmed to move autonomously. Software agents have been recognized as a promising technology to overcome issues in the mobile E-business applications [1]. They tackle major issues in wireless networks such as low bandwidth, high latency, limited battery, limited computing ability, poor display and input mechanism, expensive connection time, frequent disconnections, ubiquity, personalization and flexibility of services.

The BDI [4] models are one among the best known approaches to design intelligent agents. Cognitive agents 
use BDI model to describe the reasoning process of an agent. The BDI model provides an explicit, deliberative representation of three key mental structures of an agent: informational attitudes about the world (beliefs), motivational attitudes on what is required (desires) and deliberative commitments to act (intentions). Based on the beliefs, the set of intentions are generated and carried out to achieve the desires. Strength of the BDI model is the consistent usage of folk psychological notions similar to the way people communicate about human behavior. This motivated us to employ BDI architecture based mobile agents. This paper addresses the identification of relevant auctions based on bidder; auctioneer and network profiles by using agent technology embedded with BDI based cognition capabilities. Both static and mobile agents are employed in this work to accomplish the task of identifying the relevant auction sites. Section 1.1 covers some of the related works and section 1.2 covers the contributions of the authors.

\subsection{Related Works}

Some of the related works are as follows. In [5], a potential (bidder defined) auction is selected based on the closing time and the utility value when compared to other active auctions. By identifying the potential auction, the bidder agent ensures that the agent does not acquire more than one of the target product In [6], discussion on improving the feedback mechanism of on-line auction markets based on reputation, trust and worthiness is presented. The study in [7] develops a web assessment model from a consumer perspective where bidders continue to use an e-auction website based on bidders' satisfaction with the e-auction website in terms of user-friendliness, functionality and interactivity. The authors in [8] present analytical, computational, and empirical analyses of strategies for intelligent bid formulations in on-line auctions.

The authors in [9] discuss an intelligent agent based model for E-auction. Here the agents act together with buyers, sellers and auctioneers to assist them obtaining the best deal or at least find Nash equilibrium point. The work given in [10] expresses the bidder's perception towards identification of relevant E-auction websites in China based on trust, design and country of origin (COO). The work concluded that better design of auction sites attracts many bidders and more people trust the site. It is often preferred by many bidders as a relevant site.

In [11], a method to express the cause of bidder's choice of auction sites is presented. By understanding the bidder satisfaction the design of a bidder-friendly user interface can be fostered thus increasing the effectiveness of auction websites so that most of the bidder's are attracted to select it as relevant auction site. In [12], an agent based trust management model based on weight value for on line auctions that improves the scalability and efficiency of online auctions is presented. The methodology considers time, optimal price and trust value of the bidder into consideration.

In [13], authors propose Advertisement Based Service Discovery (ABSD) an approach to discover the web services. This work first enables the service providers using agents to dynamically and autonomously advertise semantic descriptions of available services by proposing a pay-per-click auction model. The advertisements are published after the service providers bid and win in the auctions for the space on the WWW. Then the service requesters use two levels to filter the advertised services in order to efficiently discover the eligible services. In the first level of filtration, a semantic-based mechanism for matchmaking between services requested by buyers and those advertised by sellers is applied. Services which pass first level of filtration are then considered on the second level. Here information regarding the performance of the service providers along with their bid prices in the pay-per click auction is considered. A final set of advertised services are chosen and proposed to the buyer agent as a reply to their request. The second level of matching in the aforesaid work (ABSD) is used to compare with our proposed model. The second level of matching in the aforesaid work does not consider the bidder's requirements, preferences and the constraints for identifying the services.

As per our literature survey, it is evident that the works mentioned emphasize on appearance of auction sites than on bidder requirements, preferences and constraints to identify relevant auction sites. The existing works do not discuss on the need of filtering and identifying the relevant auctions from a huge set of concurrent active auctions based on the bidder requirements and preferences.

\subsection{Our Contributions}

This paper is an extension to the work done in [14] where auction services are discovered and placed in regional gateway active auction service directory. We propose an intelligent agent based model to identify relevant set of auctions (English auctions are considered) for a mobile bidder based on bidder, auctioneers and network profiles from a set of active auctions available in the active auction service directory of regional gateway. The bidder profile contains budget, probability of winning, desperateness, and time limit to bid a product. The auctioneer profile maintains completion time, offers, average winning price, opening bid price, number of participants, bid value in concurrent active auctions and reservation price for each auction. The network profile comprises of bidder mobility and channel usage.

The model works as follows. 1) Upon arrival of a bid- 
der request, an intelligent bidder agent in the mobile device searches for matching auctions based on product in the bidder belief set. The functionality of the agent is based on Belief, Desire and Intention (BDI) cognitive architecture. 2) If matching auctions are not found in the bidder belief set, bidder agent migrates to regional gateway that maintains active auction cases (comprising of auction site and relevance factor) and searches in the case base. 3) If no match is found in case base, the agent computes a preference value for each parameter in all the profiles for all the active auctions for a requested product fetched from active auction service directory of regional gateway. 4) Relevance factor is computed by the agent for all the active auctions using the preference values of the parameters. 5) K-means clustering algorithm is employed by the agent to filter the active auctions in a hierarchical manner based on the relevance factor.

Our contributions in the paper are as follows. 1) The parameters considered for relevant auctions identification are unique and reflect the bidder requirements, constraints and preferences. 2) BDI agent with mobility feature is employed that provide autonomy, flexibility and ability to decide intelligently in dynamic situations by using belief set located in the mobile device as well as regional gateway. 3) Case based beliefs in regional gateway provides human like reasoning by offering the information about past auction cases. 4) K-means algorithm is used to improve the accuracy of auctions identification as per the specified requirements and 5) The scheme is validated by simulation results and by comparing it with ABSD. The results showed better performance than ABSD in terms of availability, scalability, winning probability, satisfaction and personalization. ABSD is chosen for comparison since it actually dealt with considered problem of relevant auction identification.

\section{Proposed Work}

This section provides description of the proposed work in terms of the network and market environment, computational models and agencies.

\subsection{Network Environment}

The network environment consists of clusters of auction servers in fixed network, regional gateways, and mobile bidders (may be sometimes auctioneers if desired) in the wireless environment. Clusters are categorized based on their physical geographical locations where each cluster consists of auction servers (AS1,ASk) hosting several auctions. The gateways are connected to the network based on the regions. Mobile bidders are in the vicinity of a wireless local area or in a cellular network. The mobile bidders in a particular region request its regional gateway for auction services. The gateway comprises of auction service directories, case base and the computational methodologies to identify the relevant auctions to participate in bidding process. An agent platform exists in all the components of network environment to facilitate agent based activities. The agent platform supports persistence, securities, communication and computing services.

\subsection{Market Environment}

The electronic marketplace consists of a number of English auctions that run concurrently on various auction servers that are connected to the WWW. Each auction house located on a server conducts multiple auctions for various products and comprises an agent platform that hosts an agency to carry out the auctions and communicate with the bidders. Each active auction maintains an E-board to display information of active auctions. The auctions have a defined start and end time which varies from one auction to another. Thus there can be multiple auctions running in parallel for a product in the market at a given time with overlapping start and end times. The bidders register themselves to fetch the relevant auctions to participate in bidding in the registration desk of the regional gateway. The service directory on the regional gateway provides the information of all active auctions for the products requested by the bidder's. The case base that acts like a cache improves the access time of the bidder. Every bidder shall have a belief set of varying size depending on the type of handheld device.

\subsection{Computational Models}

This section describes the computation of preference values of the considered parameters and clustering the auction sites based on the computed relevance factors. The bidder identifies a set of relevant auctions to participate in from among the active auctions for a time interval based on the bidder's profile, the auctioneer profile and the network profile (see section 1.2 for parameters in profiles). For each parameter a preference value is computed which lies in the range 0 to 1 , with 0 as least preferred and 1 as the most preferred.

\subsubsection{Bidder Profile}

Budget - the bidder prefers to participate in an auction if the current highest bid value is less than its allotted budget and also have maximum difference between its budget and current bid so that he has better chance to bid effectively. The preference factor is computed as follows: $p b_{1}=$ ((budget - current bid value)/budget).

Desperateness of Bidder - it indicates how deterministic a bidder (denoted as $p b_{2}$ ) in winning an item by looking at its past participation. This value is assumed to be given by either the bidder or a tool. 
Probability of winning - it is computed based on the past history of the auctions conducted for the requested item in an auction site. Let $N_{w}$ be the number of times that the bidder would have won with bid value $\mathrm{z}$ in each past auction and $\mathrm{T}_{\mathrm{a}}$ be the total number of past auctions. The preference factor $p b_{3}$ for this parameter is given by $p b_{3}=$ $N_{w} / T_{a}$. The value of $N_{w}$ is computed by the bidder and Ta is available in the service directory.

Bidder maximum time - the completion time (compt) of the active auctions for the required products available from service directory are compared with the bidders available time (avt) to compute the preference factor $\mathrm{pb}_{4}$. $p b_{4}=1-($ compt/avt $)$.

\subsubsection{Auctioneers Profile}

Auction Completion Time-the bidder may prefer to participate in the auctions that have just begun. The preference factor pal for this parameter is computed as $p a_{1}=1-(($ current time - start time $) /($ end time - start time $))$.

Offers - the bidders have higher preference to participate (denoted as $\mathrm{pa}_{2}$ ) in the sites that have offers which are announced on the auction sites e-board. It is given by $p a_{2}$ $=($ offer value $) /($ expected closing price $)$.

Average Winning Price - the bidders prefer an auction site if the average winning price (AWP) of a product in the past auctions in that site is less than or equal to the market price/assessed price (MP). It is denoted and given by $p a_{3}=$ 1- (AWP/MP). The bidder can obtain the AWP of an item at each active auction from the service directory. The market price is either obtained from the recommender or the bidders themselves assess it.

Opening Bid Price - the opening bid price (OB) influences the closing price so that the bidders prefer to participate in the auctions that have a lesser opening bid. It may be obtained from the service directory. Let $p a_{4}$ denote the preference factor for this parameter which is given as $p a_{4}=1-\mathrm{OB} / \mathrm{MP}$.

Number of Participants-bidding is rigorous if the number of bidders $(\mathrm{Nb})$ are greater than the average number of bidders $(\mathrm{ANb})$ in the past and hence bidders prefer to participate in the auctions that have fewer (lesser than average) bidders. Let $p a_{5}$ denote the preference factor based on number of bidders which is computed as $p a_{5}$ $=(\mathrm{Nb}-\mathrm{ANb}) / \mathrm{ANb}$.

Bid Value in Concurrent Auctions-bidder prefers to participate in an auction that has least current bid value (Bv). Let BVi be the bid value at the ith active auction site after predefined number of iterations or time since commencement of auctions. Let $\mathrm{V} P \mathrm{~Pa}$ be the average bid value at other concurrent auction sites at ith iteration or time. The preference factor (denoted as $p a_{6}$ ) for this parameter is given by $p a_{6}=(\mathrm{VPa}-\mathrm{Bv}) / \mathrm{VPa}$.

Reservation Price - the bidder prefers to participate in those auction sites that have the reservation price. Let $\mathrm{pa}_{7}$ be the preferential factor for this parameter which is given by $p a_{7}=1-$ (reservation price/private valuation).

Quality of Service (QoS) - the promptness in delivery of the product as per the commitment (PQ1), the availability of the bandwidth at the server end to participate satisfactorily till the completion of the process (PQ2), the complexity of the payment process (PQ3) and confidentiality \& security of bidders transactions (PQ4) determine the QoS of an auction site. These values are obtained as feedback from the participated bidders in the past. Let $p a_{8}$ denote the preference factor for QoS of an auction site which is given by $p a_{8}=\left(P Q_{1}+P Q_{2}+P Q_{3}+P Q_{4}\right) / 4$.

\subsubsection{Network Profile}

Mobility - the bidders may dispatch a mobile agent to bid on their behalf to the nearest base station before they loose connectivity. The agent may continue to send requests and receive replies from the base station. The preference factor pn1 for this parameter is given by

$\mathrm{pn}_{1}=1-$ (successful replies received/total requests for considered process).

Limited bandwidth - communication in wireless environment encounters the problem of limited bandwidth. The auctions that support agent to overcome bandwidth limitations is preferred since the bidder is free from spotty connectivity and/or no onnectivity at all. It is denoted by $\mathrm{pn}_{2}$ and is given as

$\mathrm{pn}_{2}=1-$ (successful replies received per second/total requests per second for considered process).

\subsubsection{Relevant Factor ( $r f$ ) Computation}

For all the determined parameters in all the profiles, a normalized value is computed using exponentially weighted moving average. It is given by the Equation (1). Considering the previous value will help to determine the reliability and dynamics of an auction site.

$$
r f n=\Phi r f_{c}+(1-\Phi) r f_{c-1}
$$

where $r f_{\mathrm{n}}$ is the new computed value, $r f_{\mathrm{c}}$ is the current computed value, $r f_{\mathrm{c}-1}$ is the previous value and $0 \leq \Phi \leq 1$ is a constant. $r f_{\mathrm{c}}$ is given by Equation (2).

$$
r f_{c}=\omega_{1} \zeta+\omega_{2} \psi+\omega_{3} \gamma
$$

Such that $\sum \omega_{i}=1$ for $i=1$ to $n . \omega_{i}$ is defined by the bidders. $\zeta, \Psi$ and $\gamma$ are given with respect to bidders, auctioneers and network profiles, respectively. They are defined as follows.

$\xi=\sum \rho_{j}^{b} p b_{j} \quad$ for $j=1$ to 4 and $p b_{j}$ are the bidder profile parameters, $\psi=\sum \rho_{k}^{a} p a_{k}$ for $j=1$ to 8 and $p a_{k}$ are the auctioneer profile parameters, $\gamma=\sum \rho_{o}^{n} p n_{o}$ for $n=1$ to 2 and $p n_{o}$ are the network profile parameters and $\sum \rho_{j}^{b}=1$ for $j=1$ to $1, \sum \rho_{k}^{a}=1$ for $k=1$ to $m$ and
$\sum \rho_{o}^{n}=1$ for $o=1$ to $p$.

\subsubsection{Relevant Auctions Clustering Model}

In order to classify the set of active auctions as relevant 
and non-relevant, a procedure based on K-means clustering algorithm [15] is applied. The set of active auctions with $r f$ values are given as input to the procedure and the output will be a classification of relevant and non-relevant auctions. The procedure is as follows.

1) Select random seeds as the values of the two centroids $\left(\mathrm{C}_{\mathrm{k}}: \mathrm{k}=1,2\right)$ of the clusters around which the active auctions will be scattered and the clusters do not overlap.

2) Find the average of the Euclidian distance of pf of each auction with their respective centroid in their cluster.

3) Residual sum of squares (RSS) is used to measure how well the centroids represent the members of their cluster. The squared distance of each vector from its centroid is summed to get over all $p f$.

4) The value of RSS is to be minimized by re-computing the values of the centroids in their respective cluster.

5) This procedure is repeated till minimum constant RSS is obtained.

\subsubsection{Computation of Similarity Strength}

The similarity strength normalized to 1 is computed based on the number of matching parameters between the products $(p j)$ in the bidders' $i^{\text {th }}$ request $\left(r_{i}\right)$ that did not match in the bidder belief set and the products $\left(p_{c r}\right)$ in the $\mathrm{k}^{\text {th }}$ case in the case base ck of regional gateway. The five parameters of the product to be matched are product name, specifications, bidder budget, and desperateness and bidders maximum available time. Similarity strength is a real number $j[0,1.0]$. It is incremented by 0.2 for each matching parameter with initial strength $=0$. When the strength reaches the threshold value 0.6 , it means that the product name, specifications and budget are matched.

\subsection{Agencies}

In this section, we discuss the bidder agency and the gateway agency used in the model. The main task of bidder agency located in the mobile device is to create the software agents which on behalf of the bidder will fetch and identify the relevant auctions from the set of active auctions. The gateway agency located in regional gateway provides resources for computations and hosts active auction service directory $\&$ case base. The agencies are explained as follows.

\subsubsection{Bidder Agency}

It resides in the bidder device and creates an autonomous environment to perform the entire task of fetching relevant auctions. It interacts with the bidder and the gateway agency during the entire process of identification. It creates a bidder admin agent (BAA) to control and coordinate the bidder requests, belief agent (BA) that maintains and updates the belief set that consist of $\log$ of active auction services and the bidder agent (BdA) for identification of relevant auctions. Figure 1 shows the different

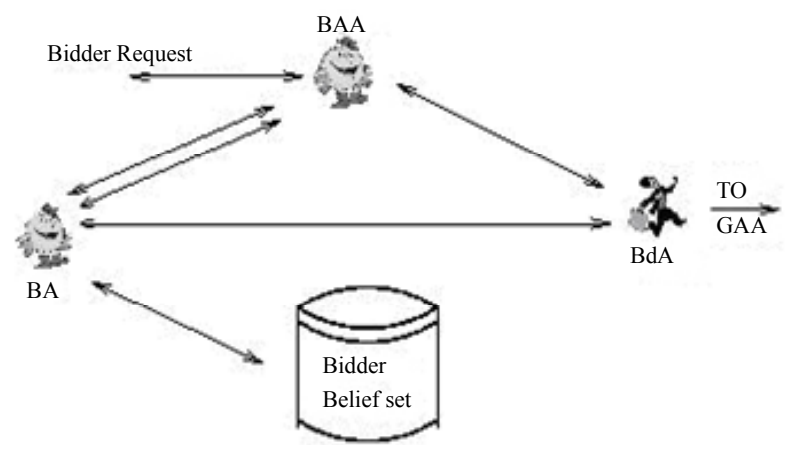

Figure 1. Bidder Agency.

components in the bidder agency.

Bidder Belief Set - is present on the bidder handheld device and consists of customized subset of auction service directory. Each record in the belief set has details similar to the service directory. The products in the belief set that have relatively poor reference by bidder are replaced with the new requested products. For example, each auction site details require $8 \mathrm{~KB}$ of memory and an item may have up to 10 auction sites. Thus total memory required for an item is $10 * 8 \mathrm{~KB}$. The belief set size may vary from $1 \mathrm{MB}$ that accommodates entries for 12 products to $4 \mathrm{MB}$ that can accommodate up to 50 products based on the handheld device. Belief set of the bidder is formulated based on initial distinct requests that depend on the size of belief set.

BAA - it is a static agent that creates, controls and coordinates all the agents in the bidder agency. It creates a unique $\mathrm{BdA}$ upon receiving a request from the bidder to act on their behalf and a BA to maintain the belief set on the bidder device. The bidder request consists of the names and specifications of the products along with the bidder constraints like the maximum available budget and time, desperateness of the bidder, bidder preferences like offers in the auction site, auction completion time, etc. It parses the bidder requests to generate the desires (required products) and loads them in the respective BdA.

$\mathrm{BA}$ - it maintains the belief set at regular intervals by identifying the BdA behavior with reference to access to the products and auction sites.

$\mathrm{BdA}$ - it is a BDI mobile agent that has the intelligence and computations (intentions) required to carry out the desires of the bidder's. Figure 2 shows the BDI based bidder agent.

- Desires-BdA receives the desires from the BAA. The desires may be either homogeneous or heterogeneous. Homogeneous are the ones that have similar requirements like the same products with different specifications or belong to same category (mobile devices, books history, jewellery, crockery, etc.) of products, otherwise they are heterogeneous. 
- Belief set-It has two belief sets, one in the bidder device and the other in the regional gateway (called as case base). Bidder belief set is as explained above. The corresponding details for the requested products are fetched from the service directory and filled in the bidder belief set. The auction details for the products in the belief set are also updated when they receive the active alerts or advertisements. The popularity factor of the items /products are computed at regular frequency as ratio of frequency of referring each item within the belief set and the number of requests for a predefined time. The products in the belief set that have relatively poor popularity are replaced with the new requested products at regular frequency. Case-base is explained in gateway agency.

- Intentions module - three phases of the intention module are generation of set of intentions (library of plans), identifying the appropriate intention and execution [16]. The Intention generation phase receives the desires and accordingly generates the intentions. Three possible intentions of the BdA are as follows. 1) to search for the requested products/desires in the local belief set in the bidder device. It may find matches for all the requested products or only for few of them. 2) For the products that did not find a match, BdA alters its intentions and migrates to the gateway along with the remaining products list/desires to find a match in the case base. 3) If it does not find satisfactory matching cases, then the BdA will fetch the active auctions from the service directory and then compute $r f$ of those active auctions using the computation model and clusters relevant auctions using relevant auctions clustering model.

The decision making phase decides which of the three intentions to be selected for the bidders' desires. The execution phase executes the identified intention appropriately. These set of relevant auctions along with the desires may be stored as a new case in the case base.

\subsubsection{Gateway Agency}

This agency in the regional gateway comprises of Gateway Admin Agent (GAA), Registration agent (RA), Directory agent (DA) and Case agent(CA). It also hosts and maintains service directory and case base. Figure 3 shows the different agents and databases in the gateway agency.

Service directory-Service directories store the details of the auction services in a distributed tree structure as given in [14] and [17]. Services in a server i.e., on all the leaf nodes are stored in a form of a template which consists of the item name with specifications, list ofauctions, details of each auction like start and end time, average winning price in the recent past, current bid value, offers if any, opening bid price, number of registered participants, reservation price and popularity factor of each auction site. Popularity factor of an item gives the number

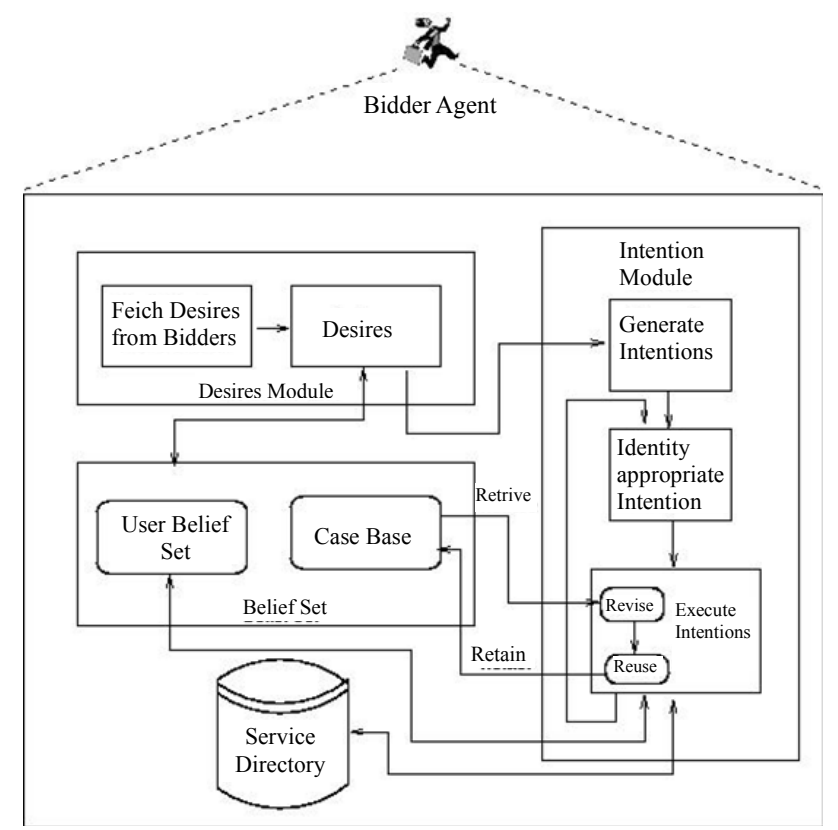

Figure 2. Mobile BDI Bidder agent.

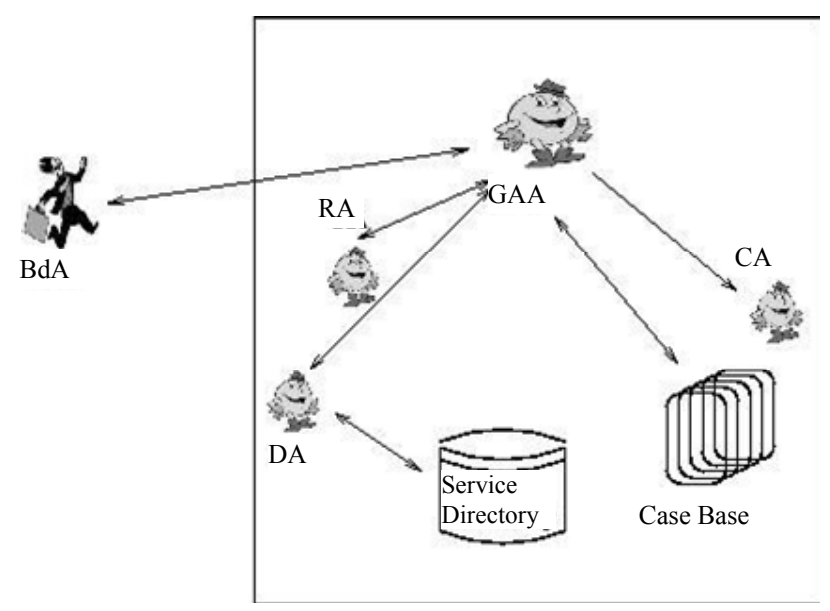

Figure 3. Gateway agency.

of times it has been referred for searching its auction site and also the value of the factor is increased based on the bidder feedback.

Case base-A case is a contextualized piece of knowledge representing the experience. The Case base consists of $\log$ of past cases. BdA after reaching the regional gateway, registers itself and seeks permission to communicate with local agents and use the resources like processor and memory. It continues to execute its second option of intentions using Case Based Reasoning (CBR) given in [18] to search the matching cases in the case base. The CBR is embedded within BDI architecture to achieve the desires. A request from the bidder is considered as new case and all matching cases are retrieved from the 
case base. For the products in the new case, the best matching products from the matching cases are combined and retained after reusing. In the proposed work, the case base is considered as a belief set that has collection of cases and resides in the gateway and is common to all the bidders. The cases in the casebase are categorized into clusters as shown in Figure 4.

The popularity factor of a case is the ratio of number of times it was retrieved to the total number of requests. The case with higher popularity factor is placed in higher level in the hierarchy within a cluster. This method of organization of the cases supports in retrieving the cases accurately and effectively.

Initially, the cases are generated by storing responses for first few requests by explicitly collecting it from service directory and computing the $r f$ of each active auction and builds a case index file. When a request (item name with specifications, bidder constraints and preferences) arrives, the case index file (Table 1) is searched for matching product name and in turn fetches the offsets of the corresponding cluster and cases within it. For all the matching cases in the identified cluster, the similarity strength between the products in the bidder request and

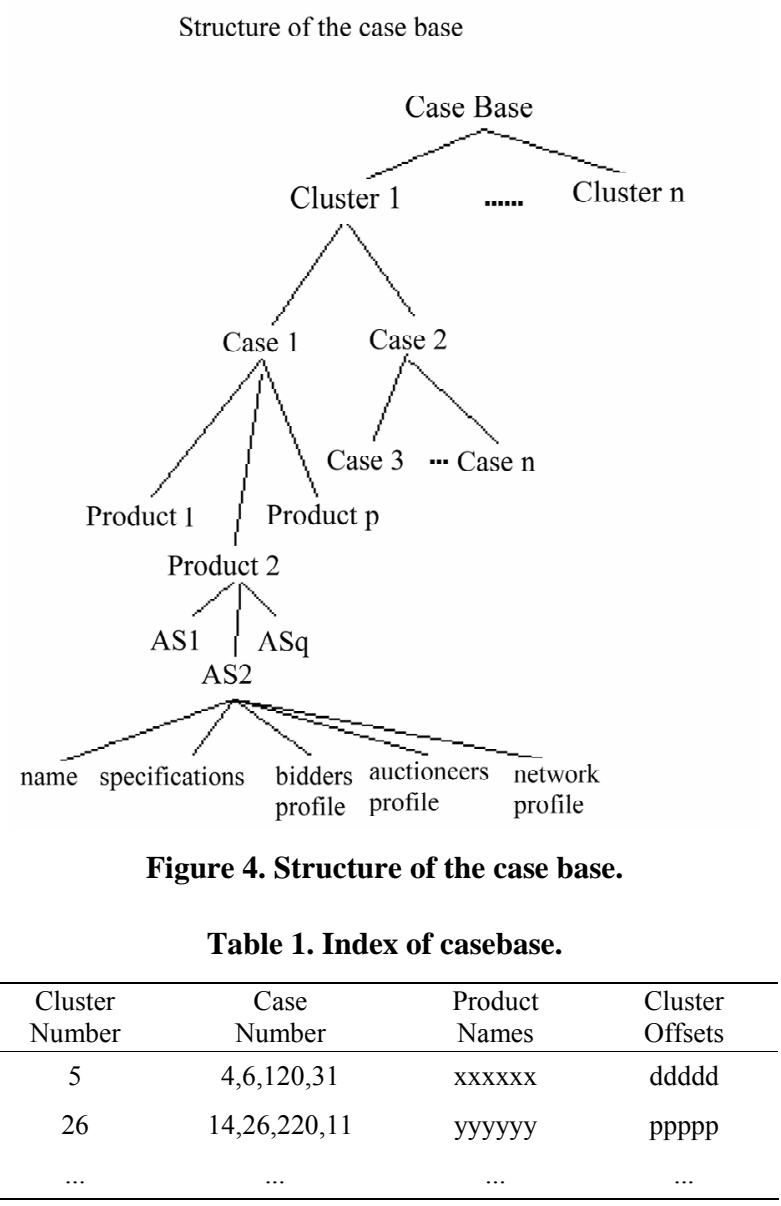

the matching cases is computed as given in computational model. The cases/products that have relatively better similarity strength and the popularity factor are retrieved and reused.

For the requests where the case base does not contain any matching cases, $r f$ is computed as given in computational model after fetching information from active auction service directory and it revises and updates the case base with a new case. The clusters in case base are revised gradually as and how the cases are referred. The replacement/retaining policy uses the human concept of forgetting/replacing the cases in the case base according to their age and popularity. A case is not popular if it is not referred for a long duration of time. The log of previously executed desires in the form of cases which are not specific to a particular bidder are accumulated.

GAA - is a static agent which is responsible for creating several agents that assist in identifying relevant auctions as per the bidder requests and filtering them as per the bidder preferences. It creates, controls and coordinates all the agents residing on the gateway. It creates a RA that registers the $\mathrm{BdA}, \mathrm{a} \mathrm{DA}$ and $\mathrm{CA}$ to maintain, update and access service directory and case bases. After it receives a request from $\mathrm{BdA}$, it directs the RA to register the bidder request. The BdA after seeking the permission from GAA to access the resources uses BDI based CBR to retrieve the matching cases and identify the relevant auctions among them. If $\mathrm{BdA}$ does not find products matching the desires it invokes the DA to fetch the set of active auctions from the service directory that match the bidder preferences for the products mentioned by the BdA and compute relevance factor for each active auctions and cluster them to get the desired set of relevant auctions.

RA - is a static agent created by the GAA. It registers every BdA and deals with the issues relating to authentication of BdAs and issues permission to interact with local agents and use resources on the gateway to fetch the required information.

DA - is a static agent created by the GAA to fetch the auction services from the service directory for the products requested by the BdA. It returns the list of active auctions along with the auctioneer profile parameters of the products requested by the BdA to participate in bidding.

CA - is a static agent created by the GAA to retrieve and maintain cases in the case base. It searches the matching cases in the case base against the requested products sent by the $\mathrm{BdA}$. The computation of matching strength is discussed in computational model. The similarity degree is computed for each item mentioned in the BdA with all the matching cases and then combines the best matching cases for all the products and returns them to BdA. This will also be updated in the case base. 


\subsubsection{Agent Interactions}

Figure 5 represents the agent interaction sequence of the proposed scheme. The sequence is given as follows. 1) The BAA creates a BdA based on the request of the bidder to discover the auction services and identify the relevant auctions on behalf of the bidder.(2) The BdA communicates with the BA to find if the local belief set meets the bidder's requirements. 3) If the services are available either partially or completely on the local belief set they are returned to the BAA. 4) For the remaining products, the $\mathrm{BdA}$ migrates to the regional gateway and communicates to the GAA to direct RA to register it and permit it to interact with the agents on the gateway to obtain the required services and resources. 5) RA communicates and registers the BdA. 6) The BdA communicates to GAA the requirement of auction services for the products requested by its bidder. 7) The GAA directs the CA to search for the matching cases in the case base. 8) The CA returns the matching cases for all or subset of the requested products to the BdA. 9) For the remaining products the AdA requests the GAA to direct the DA to fetch the active auctions from the global service directory. 10) The DA returns the set of active auctions to the BdA. 11) The BdA further requests GAA for computing resources and memory to compute the $r f$ of the active auctions. 12) The BdA performs the computations to identify the relevant factors of the active auctions and classifies them as relevant and non-relevant. 13)The $\mathrm{BdA}$ returns to UAA the list of relevant auctions to participate and 14) BdA destroys itself.

\subsubsection{Advantages and Limitations}

The advantages of the proposal are as follows: 1) Autonomy of the identification of the relevant active auction sites is achieved. 2) The relevant auctions are identified for each individual bidder based on their requirements, preferences and constraints. 3) It reduces the search space of the bidder due to which the average response time is reduced. 4) The probability of winning

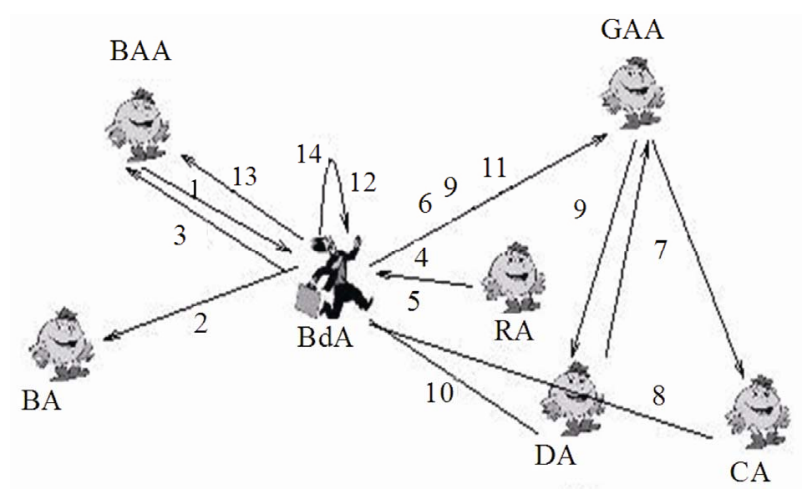

Figure 5. Agent interactions. with better satisfaction is achieved and 5) Accurate matching of services for the bidder's request is achieved. The limitations of the proposal are as follows: 1) Case base is constructed based on bidders' requests rather than using machine learning techniques. 2) The cases in the case base are deleted based on the age and its utility and 3) Maintenance of case base and belief set is an overhead.

\section{Simulation Model}

The proposed model has been simulated in various network scenarios. Simulation environment for the proposed work comprises of three models namely network model, market model and bidder model. The models are described as follows.

Network Model - the network considered for simulation is combination of both wired and wireless environment. It consists of Nc clusters, each cluster consisting of $\mathrm{Nk}$ auction servers hosting $\mathrm{Nu}$ auctions in a fixed network and $\mathrm{Rg}$ regional gateways hosting $\mathrm{Nm}$ service directories. Ns servers (a subset of $\mathrm{Nk}$ ) are connected to the gateway within the region. The number of mobile bidders and mobile auctioneers are $\mathrm{Np}$ and $\mathrm{Na}$, respectively.

Market model - the simulated virtual market place consists of Naa number of active auctions offering $S$ auction services in $\mathrm{Nk}$ auction servers. Naa is subset of $\mathrm{Nu}$ since at a given point of time $t$ not all auctions are active. The $\mathrm{S}$ auction services are fetched and stored in auction service directory. The number of active auctions and all the fields in a service are generated randomly. The market place is flexible and can be configured to run any number of auctions. Each auction auctions $\mathrm{Pk}$ products. Every active auction maintains an E-board that maintains the details of current active auctions. The relevant set of auctions $\mathrm{Nr}$ are identified based on bidder's requirements from active auction service directories.

Bidder Model - the bidders use handheld device of different specifications and hence the belief set size of bidder's vary from $1 \mathrm{MB}$ to $4 \mathrm{MB}$ that can store details of 12 products to 50 products that are auctioned. Each item stores the details and specification of products and about 10 relevant auction items. The mobile bidders are moving at a speed $x \mathrm{Km} / \mathrm{hr}$ and the bandwidth available is bMBps. At any considered time window, a set of $\mathrm{r}$ requests for $i$ products received from ' $\mathrm{Np}$ ' bidders are generated randomly. Such 100 sets of requests are generated. All the fields of a request are generated as a random number.

\subsection{Simulation Procedure}

To illustrate some results of the simulation, following data is considered: $N_{c}=1, R_{g}=1, N_{k}=2$ to $150, N_{u}=1$ to 500 , $N_{m}=2-8, N_{s}=2$ to $8, N_{p}=1$ to $500, N_{a} 1$ to $200, S=1000$ to $200,000, N_{a a}=5$ to $500, P_{k}=200$ to $1000, N_{r}=1$ to 100 , $r=1$ to $500, i=1$ to 500 and $x=20$ to 180 and $b=2$ to 8 , 
In bidder request the product name $=1$ to 100 , specifications of the product $=1$ to 100 , budget $=500$ to 10,000 , desperateness of bidder $=0.0$ to 1.0 , bidder available time $=1$ to 8 hours. In the service directory the product name $=$ 1 to 1000 , specifications $=1$ to 100 , start time $=2$ to 5 , end time $=12$, avg win price 1000 to 9000 , open bid $=100$ to 500 , current bid $=100$ to 9000 , number of participants $=5$ to 100 , offers $=100$ to 3000 and reservation price $=500$ to 1000.

The simulation procedure is as follows. 1) Fetch the bidders requests. 2) Formulate the bidder belief set and case base. 3) Search the relevant auctions in the bidder belief set and/or case base. 4) If not successful in above search then fetch the active auctions information from service directory. 5) Apply the proposed computational model to identify the relevant auctions from the active auctions as per bidder requirements and preferences and 6) Compute the performance of the model.

\subsection{Performance Measures}

The following performance measures are considered in simulation. Availability of Services-is the percent of relevant auction services available in bidder belief set, case base and service directory for the homogeneous and heterogeneous requests of bidders. Average Response Time - is the average time taken to identify the relevant auctions for set of bidder's requests Personalization - it is the percent of matching between the bidders' requirements and specification of products in the requests with the products in the discovered auction services. Winning Probability with Satisfaction - it is the probability of winning using the relevant auction set. Successful Replies - it is defined as percentage of successful replies per unit of time for a set of bidders request with respect to the bidders mobility during the process of discovering the services. Agent Overhead - it is the additional code, data and state of the agent that acquires the communication channel and migrates to the server. Overhead of Computations - it is the number of computations executed for computing the relevant factor of all the active auctions for requested products in identifying the relevant auctions in the presence and absence of bidder belief set and case base.

\section{Results}

In this section, the results obtained with proposed work is discussed. The simulation is carried on core 2 duo machine using ' $\mathrm{C}$ ' language. The analysis of the performance parameters are given in this section.

Analysis of Availability of Services.

It may be observed from the Figure 6 that the percent of matching cases in the case base improves with increase in number of requests. This is due to the increase in the

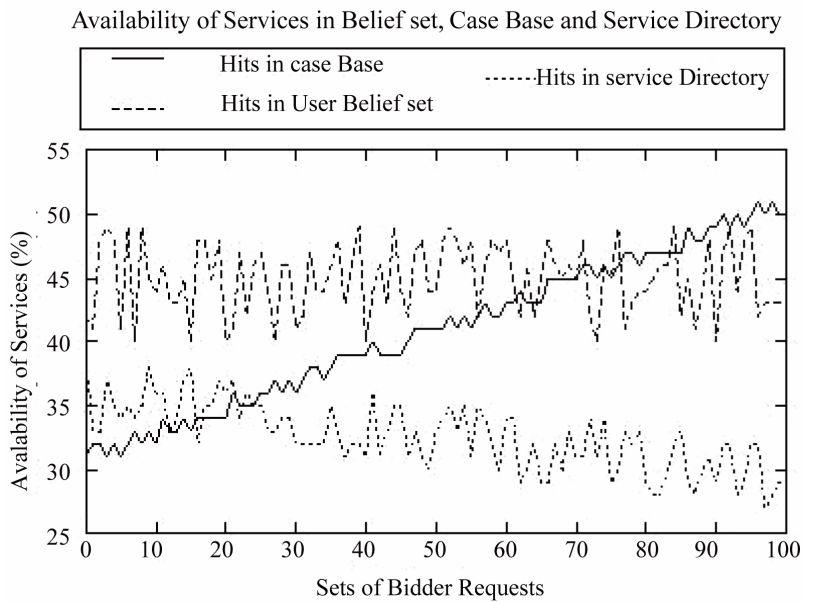

Figure 6. Availability of services vs. sets of bidder requests.

number of cases in the case base while the hits in bidder belief set has not varied much with the increase in number of requests. The hits in the service directory reduce with the increase in the hits in case base since the remaining requests are served in the service directory. It can be concluded that ninety eight to ninety nine percent of the bidder requests are replied either from belief set or case base or from service directory. More than sixty percent of the requests are processed either in bidder belief set or in case base which improves the access time and in turn the efficiency of the proposed model.

Analysis of Average Response Time-Figure 7 shows that the response time is better when the product is found in bidder belief set or case base even with increase in number of requests. Even though the access time in case base increases with increase in its size and number of hits in it, the response time is still lesser than the approach of directly searching the service directory for all the requests and then computing the relevance factors of all active auctions. The average response time in ABSD approach is higher than the proposed model. This is due to the fact that the requests have to wait till the auctions are announced and commenced in ABSD approach.

Personalization-Figure 8 shows the percent of personalization (matching as per requirements) of active auctions for the common products as required by different bidder's using BDI agent and ABSD approaches. The percentage of personalization of common products as viewed by each bidder using different approaches is different. For example, consider product 4, the percent of personalization as viewed by bidder 1, 2 and 3 using proposed approach is 100,90 and 85 percent, and using ABSD is 8,7 and 10 percent. The percent of personalization of common products using either proposed approach or ABSD varies from bidder to bidder. The variation in computing the percent of personalization using proposed 
Average Response time

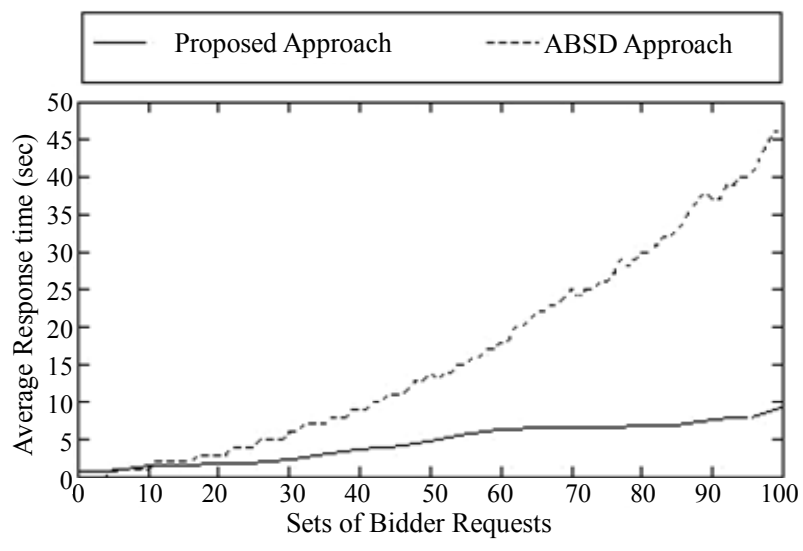

Figure 7. Average response time vs. sets of bidder requests.

Personalization of Active Auctions

\begin{tabular}{|ll|}
\hline - . C Customer 1 - proposed approach & - -.-Customer 1 - ABSD \\
- - - Customer 3 - proposed approach & - - - Customer 2 - ABSD \\
\hline
\end{tabular}

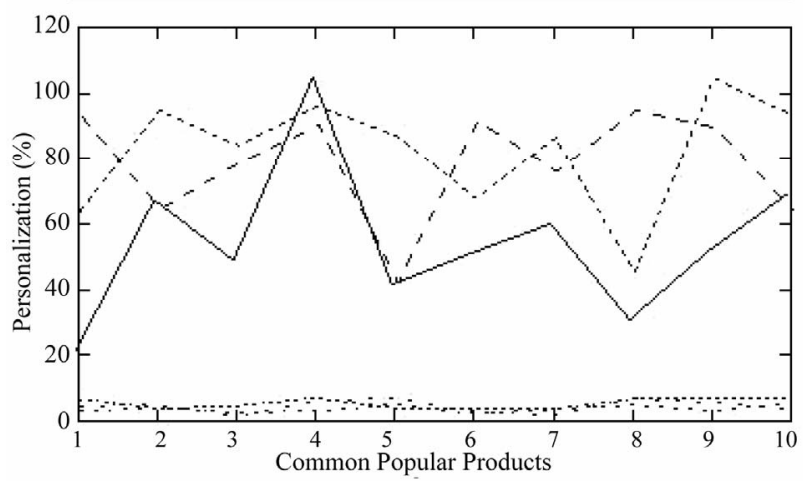

Figure 8. \% of Personalization of auction sites vs. popular products.

approach is higher than when using ABSD approach. This is because in proposed approach, the personalization is defined by individual bidder as per their requirements which are computed as $r f$. This depicts individual customization. In ABSD approach, the variation in percent of personalization of auctions for same product and by different bidder's is not much. This shows that customization is weak in ABSD approach.

Winning Probability with Satisfaction - The bidder has a better probability of winning and with satisfaction by participating only in the set of relevant (personalized) auctions than participating in the larger set of active auctions which is depicted in the Figure 9. This is because the probability of winning with satisfaction increases as the set of relevant auctions are less and satisfy most of the bidder's requirements than the general set of active auctions. The probability of winning with satisfaction using ABSD is lesser than the proposed approach.
Analysis of Successful Replies - Figure 10 shows the effect of mobility of bidder on percent of successful replies he receives for various loads of the server. It may be observed that as the speed of the bidder increases the \% of replies received reduces more due to the handoff effect then due to disconnections or spotty connectivity.

Analysis of Agent Overhead - Figure 11 shows the agent transmission time for different availability of network bandwidth. It may be observed that the agent transmission time is increased with increase in bidders and the requests from the bidders. If network bandwidth is high, agents can be transferred faster to the servers. A trade off between the bandwidth utilized by the agent and the intelligent task (identification of relevant auctions) carried out by it may be considered.

\section{Conclusions}

The proposed model reduces the burden of the bidder

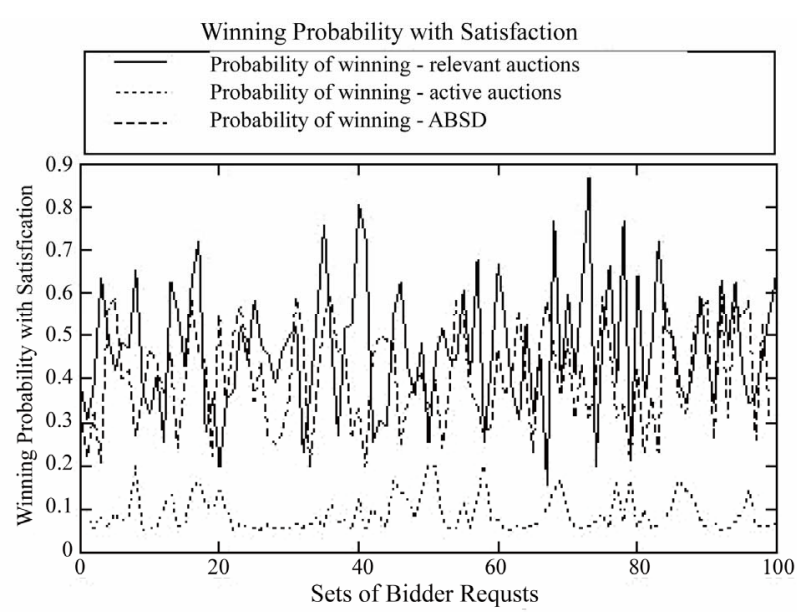

Figure 9. Winning probability with satisfaction vs. sets of bidder requests.

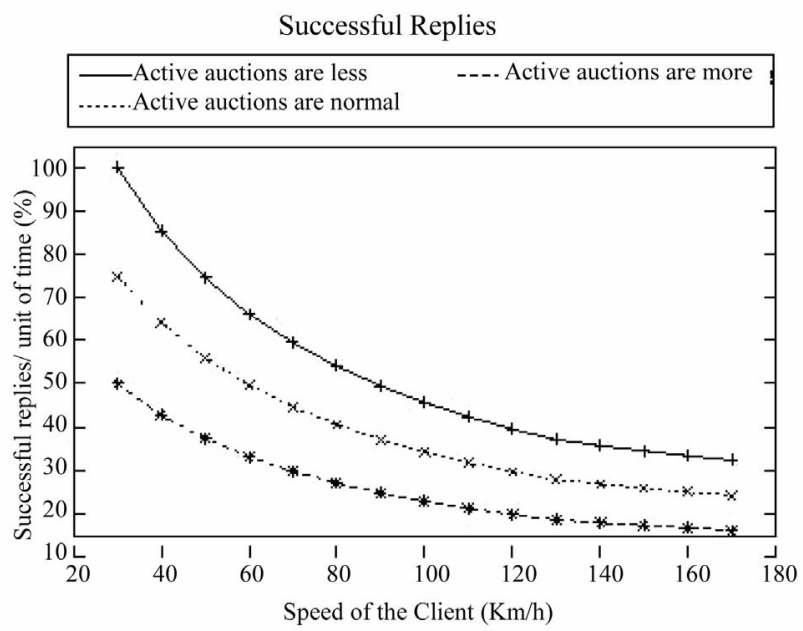

Figure 10. Successful replies vs. speed of the bidder $(\mathrm{km} / \mathrm{h})$. 


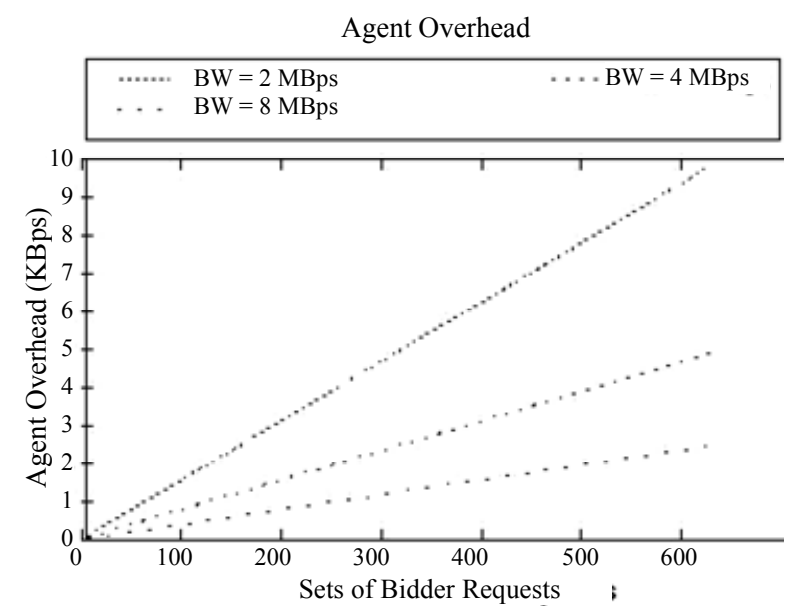

Figure 11. Agent overhead vs. sets of bidder requests.

and provides intelligent assistance to select relevant set of auctions to participate by using a cognitive agent based model dependent on beliefs. The relevant auctions match the bidder requirements and preferences to the maximum extent increasing the probability of winning with better satisfaction level. The proposed model can be part of any bidding mechanism that facilitates the bidder to participate in the relevant auctions depending on bidder profiles, network profiles and auction profiles by using multiple collaborative bidding agents. The work can be extended to include some machine learning techniques to develop the beliefs in the bidder and auction servers.

\section{REFERENCES}

[1] U. Varshney and R. Vetter, "Mobile Commerce: Framework, Applications and Networking Support Mobile Networks and Applications," Mobile Netorks and Applications, Vol. 8, No. 3, June 2006, pp. 185-198.

[2] A. Outtagarts, "Mobile Agent Based Applications: A Survey," International Journal of Computer Science and Network Security, Vol. 9, No. 11, September 2009, pp. 411-415.

[3] N. Sidnal and S. S. Manvi, "Are Mobile Agents Suitable for Mobile Commerce," Proceedings of IEEE National Conference on Information Communication Convergence, IEEE Computer Society, Chennai, December 2006, pp. $342-422$

[4] A. Rao and P. M. Georgeff, "Modeling Rational Agents within a BDI Architecture," Proceedings of the 2nd International Conference on Principles of Knowledge Representation and Reasoning (KR91), Morgan Kaufmann, March 1991, pp. 473-484.

[5] P. Anthony and N. Jennings, "Developing a Bidding Agent for Multiple Heterogeneous Auctions," ACM Transactions on Internet Technology, Vol. 3, No. 3, August 2003, pp. 185-217. doi:10.1145/857166.857167
[6] F. Ling, "Trust and Rebates: How Online Auction Markets Can Improve Their Feedback Mechanisms," Journal of Economics and Management Strategy, Vol. 19, No. 2, May 2010, pp. 303-331. doi:10.1111/j.1530-9134.2010.00253.x

[7] J. Lu, L. Wang, C. Yu and J. Wu, "E-Auction Web Assessment Model in China," Journal of Electronic Commerce research, Vol. 9, No. 3, September 2009, pp. 123131.

[8] G. Adomavicius, A. Gupta and D. Zhdanov, "Designing Intelligent Software Agents for Auctions with Limited Information Feedback," Journal of Information Systems Research, Vol. 20, No. 4, December 2009, pp. 507-526.

[9] A. C. B. Garcia, A. Lopes and C. Bentes, "Electronic Auction with Autonomous Intelligent Agents: Finding Opportunities by Being There," Inteligencia Artificial Iberoamerican Journal of AI, Vol. 1, No. 13, March 2007, pp. 45-52.

[10] Z. Huang and M. Dai, "Users' Selection of e Auction Websites in China: The Effects of Design, Trust and Country of Origin," Issues in Information Systems, Vol. 7, No. 2, February 2006, pp. 151-163.

[11] P. L. To, C. C. Liu and C.-Y. Chen, "Online Auction Effectiveness: Optimal Selling Strategies for Online Aucton Market," Proceedings of 2008 Pacific Asia Conference on Informaton Systems, March 2008, pp. 184-195.

[12] E. Sathiyamoorthy, N. Ch. S. N. Iyenger and V. Ramachandran, "Agent Based Trust Management Model Based on Weight Value Model for Online Auctions," International Journal of Network Security and Its Applications, Vol. 1, No. 3, October 2009, pp. 15-31.

[13] P. Vedran, T. Krunoslav and J. Gordan, "An Auction Based Semantic Service Discovery Mode for e-Commerce Applications," Proceedings of OTM Workshops 2006, Springer Verlag Heidelberg, New York, December 2008, pp. 97-106.

[14] N. Sidnal and S. S. Manvi, "Auction Service Discovery Model an Agent Based Approach," In Proceedings of II International conference on multimedia and Ubiquitous Engineering (MUE. 2009), IEEE, 4-6 June 2009, pp. $573-$ 584.

[15] K. Thapas and N. Netanyahu, "An Efficient k-Means Clustering Algorithm: Analysis and Implementation," IEEE Transactions on Pattern Analysis and Machine Intelligence, Vol. 24, No. 7, July 1998, pp. 85-98.

[16] M. E. Bratman, "Intentions, Plans and Practical Reasoning," Harvard University press, Cambridge, 1987.

[17] S. Manvi and P. Venkatraman, "An Intelligent Product Information Presentation in e-Commerce," Electronic Commerce Research and Applications, Vol. 4, No. 3, July 2005, pp. 385-398. doi:10.1016/j.elerap.2005.01.001

[18] J. L. Kolodner, "An Introduction to Case Base Reasoning," Artificial Intelligence Review, Vol. 6, No. 3, September 1992, pp. 3-34. doi:10.1007/BF00155578 